\title{
STABILITY OF PLANE COUETTE FLOW FOR HIGH REYNOLDS NUMBER
}

\author{
G. B. DAVIS and A. G. MORRIS
}

(Received 8 September 1981; revised 10 March 1982)

\begin{abstract}
Experimental evidence shows that plane Couette flow becomes unstable when the Reynolds number $R$ reaches certain critical values. Linear stability theory does not predict these observations and has been unable to locate these instabilities. A Chebyshev/QR numerical technique is used to investigate much higher values of $R$ than those previously tested. In particular, values of $R$ up to $10^{8}$ are confidently tested, whereas previously values of $R$ up to only $2 \times 10^{4}$ have been considered.
\end{abstract}

\section{Introduction}

The stability of plane Couette flow has been a subject of intensive theoretical and experimental research for over three quarters of a century. The question of whether plane Couette flow is always stable to infinitesimal disturbances is still unresolved.

Plane Couette flow occurs when the flow velocity depends linearly on the transverse coordinate and is the simplest flow compatible with the Navier Stokes equations. We consider a viscous, incompressible, isotropic fluid between two infinitely long parallel planes at a constant distance apart and such that both planes move tangentially in opposite directions at a constant speed. In dimensionless variables, let the planes be positioned at $y= \pm 1$ and the Couette flow have a velocity profile given by $U(y)=y$.

Squire [17] considered theoretically the three dimensional case and showed, for disturbances periodic in the $z$ direction, that a two dimensional disturbance gives

\footnotetext{
' Department of Mathematics, The University of Wollongong, Wollongong, N.S.W. 2500.

(C) Copyright Australian Mathematical Society 1983
} 
a smaller critical Reynolds number $R_{c}$ than a three dimensional disturbance. Therefore Squire's Theorem allows us to consider the simpler two dimensional case.

Upon integration of the two dimensional continuity equation we obtain solutions in terms of the stream function $\psi(x, y, t)$. The perturbed form of the vorticity equation (as derived from the Navier Stokes equations) is linear in $\psi$ with coefficients independent of $x$ and $t$. We take the two dimensional disturbance to be of the form

$$
\psi(x, y, t)=\phi(y) e^{i a(x-c t)}
$$

where $\phi \equiv \phi(y)$ is the amplitude factor of the disturbance and is also the eigenfunction of the problem which is complex valued and depends only on $y, \alpha$ is the streamwise wavenumber, considered real and positive (complex values for $\alpha$ are to be considered at a later date), and $c=c_{r}+i c_{1}$ is the complex speed of propagation of the disturbance and is the eigenvalue of the problem.

When we superimpose an infinitesimal two dimensional disturbance of the form given in equation (1) upon the flow and by neglecting quadratic terms, we obtain the fourth order dimensionless Orr-Sommerfeld differential equation,

$$
\phi^{\prime \prime \prime \prime}-2 \alpha^{2} \phi^{\prime \prime}+\alpha^{4} \phi-i \alpha R(y-c)\left(\phi^{\prime \prime}-\alpha^{2} \phi\right)=0
$$

with boundary conditions

$$
\phi=\frac{d \phi}{d y}=0 \text { at } y= \pm 1,
$$

where $R$ is the Reynolds number which depends on $U_{0}$, the velocity of the planes, $d$, a representative linear dimension of the system, $\mu$, the viscosity and $\rho$, the density and is given by $R=\rho u_{0} d / \mu$ which is constant

It can be seen from the form of our disturbance (1), that if $c_{t}=\operatorname{Im}(c)<0$ then the disturbance will decay away with time and allow the main flow to remain stable. However if $c_{t}>0$ then the disturbances will increase and cause the onset of a growing instability. For increasing values of $\alpha R$ the initial shear flow is expected to become turbulent and therefore we would anticipate that $c_{i}$ should become positive. Turbulence in parallel shear flows has been observed experimentally by Robertson [16] and Reichardt [15] for moderate values of $\alpha R$ in the range from 600 to 1450. Theoretically however, Couette flow has always been found to be stable, that is $c_{i}<0$, for all $\alpha R$ so far considered. In Table 1 we give a summary of the values of $\alpha$ and $\alpha R$ investigated to date by various authors.

Several numerical techniques have been employed to solve particular cases of the general Orr-Sommerfeld equation. For plane Poiseuille flow Thomas [20] applied a finite difference scheme, while Orszag [13] coupled a Chebyshev 
TABLE $\}$. Values of $\alpha, \alpha R$ and maximum values of $R$ considered by various authors

\begin{tabular}{|l|c|c|c|}
\hline \multicolumn{1}{|c|}{ Author } & $\alpha$ & $\alpha R$ & Maximum $R$ \\
\hline Hopf (1914) & $0-4.64$ & $0.125-12.5$ & $\sim 5.4$ \\
Gallagher and Mercer (1962) & $0-8$ & $1-300$ & 300 \\
& 2 & $1-10^{3}$ & 500 \\
Deardorff (1963) & $0-24$ & $1-3.1 \times 10^{4}$ & 1430 \\
Lee and Reynolds (1967) & 0.5 .2 .0 & $10-200$ & 200 \\
Ellingsen et al.(1970) & $0.5,1.0$ & $10^{3}-10^{4}$ & $2 \times 10^{4}$ \\
*Davey (1973) & $0-100$ & $0-10^{5}$ & \\
Present Work & $0-4.64$ & $0.125-10^{4}$ & $10^{4}$ \\
& 2 & $1-2 \times 10^{5}$ & $10^{5}$ \\
& $10^{-4}-10^{3}$ & $10^{4}$ & $10^{8}$ \\
\hline
\end{tabular}

* Although not given explicitly in his paper Davey (1973) states that these values were considered by him.

polynomial approximation with the $\mathrm{QR}$ algorithm of Wilkinson [23]. For the particular case of a Blasius boundary layer velocity profile, Jordinson [9] and Osborne [14] programmed an iterative scheme based on the finite difference approximations associated with the Orr-Sommerfeld equation. To calculate the neutral curve for the Blasius profile, van Stijn and van de Vooren [21] applied a shooting procedure together with a method of order reduction. For plane Couette flow, as given in equation (2), Grohne [7], Hopf [8] and Ellingsen, Gjevik and Palm [3] employed asymptotic expansions, [3] and [8] considering expansions in terms of Bessel and Hankel functions. Gallagher and Mercer [5] and Lee and Reynolds [10] applied variational techniques with differing approximating functions. Deardorff [2] coupled a finite difference scheme with a trial and error numerical method, and Davey [1] applied a parallel shooting procedure with 'complete' orthonormalization.

In this paper the calculation of the eigenvalues is performed using a method of Chebyshev polynomial approximation developed by Morris [11] and Morris and Horner [12] coupled with the QR algorithm of Francis [4]. Morris and Horner [12] comment on the accuracy of this method by comparing Chebyshev series solutions with known results. In [11], various forms of the Orr-Sommerfeld equation are treated and results compared with those of previous authors; and in particular for Couette flow a comparison is made of standard differential equation solutions with finite difference method results obtained in [14]. Orszag [13] shows that solving the Orr-Sommerfeld equation numerically using expansions in Chebyshev polynomials coupled with the $\mathrm{QR}$ algorithm gives results of "great accuracy... very economically". 


\section{Chebyshev /QR technique}

The method of Chebyshev polynomial approximations employed in this work is taken from the paper by Morris and Horner [12]. Following the notation of Morris and Horner a brief summary of the Chebyshev series method is given.

They considered general problems of the form

$$
\sum_{i=0}^{4} p_{i}(y, \xi) \frac{d^{\prime} \phi}{d y^{t}}=0, \quad \phi( \pm 1)=\phi^{(1)}( \pm 1)=0,
$$

where $p_{i}(y, \xi)$ are polynomials at most quadratic in $y$ and linear in an eigenvalue $\xi$. It is assumed that the solution $\phi(y)$ can be written as an infinite series of Chebyshev polynomials in the form

$$
\phi(y)=\sum_{k=0}^{\infty} a_{k} T_{k}(y)=\frac{1}{2} a_{0}+a_{1} T_{1}(y)+a_{2} T_{2}(y)+\cdots
$$

where $T_{k}(y)$ is the Chebyshev polynomial of the first kind of degree $k$, given by $T_{k}(y)=\cos \left(k \cos ^{-1} y\right), a_{k}$ is independent of $y$ but dependent on the eigenvalue $\xi$, and $\Sigma^{\prime}$ denotes a summation involving $\frac{1}{2} a_{0}$ rather than $a_{0}$. The derivatives, $\phi^{(i)}(y),(i=0,1,2,3,4)$ can be written

$$
\phi^{(l)}(y)=\sum_{k=0}^{\infty} a_{k}^{(\prime)} T_{k}(y)
$$

with $a_{k}^{(0)} \equiv a_{k}$ and $\phi^{(0)}(y) \equiv \phi(y)$.

On the substitution of (4) into (3) a set of results can be obtained in the general form

$$
\sum_{l=k-m}^{k+m}\left\{\sum_{j=0}^{12} c_{j} w_{l j}\right\} a_{l}=0, \quad k=s, s+r, s+2 r, \ldots
$$

The relevant coefficients $w_{l}$ for the differential equation governing Couette flow can be found in Table 1 of [12]. Since the coefficients $c_{i}$ are linear functions of the eigenvalue $\xi$, the eigenvalue problem then reduces to solving

$$
[A-\xi B] \mathbf{a}=\mathbf{0} \text {. }
$$

This resulting matrix equation is then solved using the $\mathrm{QR}$ algorithm of [4].

All calculations carried out were performed to double precision accuracy using complex valued variables and functions on the UNIVAC 1106 computer at the University of Wollongong. Eigenvalue results are stated to six significant figure accuracy throughout. The order $N$ of the matrix necessary for convergence varied from 15 to 100 depending on the values of $\alpha$ and $\alpha R$. 


\section{Results}

The eigenvalue $\lambda_{1}^{\prime}$ tabulated in Tables 2, 3,5 and 6 is defined as in Gallagher and Mercer [5] as $\lambda_{1}^{\prime}=\left(4 \alpha / \pi^{2}\right)(i R c-\alpha)$. In Tables 2, 3 and 4 we compare the results obtained using the Chebyshev/QR technique with those of Hopf [8], Gallagher and Mercer [5], Lee and Reynolds [10] and Ellingsen, Gjevik and Palm [3]. These are tabulated firstly to substantiate previous efforts to obtain the eigenvalues of the Orr-Sommerfeld equation, and secondly to compare results obtained by such widely varying techniques as used by these other authors. The largest discrepancy in results observed, occurs in the third significant figure (excluding [8]), and so we consider, with confidence, higher values of $\alpha$ and $R$ using the Chebyshev series/QR algorithm method (also bearing in mind the comments of [11], [12] and [13]).

In Table 5 we show results for $\alpha R$ in excess of not only any previous authors' attempts, but also values of $\alpha$ and $R$ in excess of the experimental values of the

TABle 2. Hopf (1914). Gallagher and Mercer (1962) and Chebyshev results for $\operatorname{Re}\left(\lambda_{1}^{\prime}\right)$

\begin{tabular}{|c|l|c|c|c|}
\hline \multicolumn{2}{|c|}{} & \multicolumn{3}{|c|}{$\operatorname{Re}\left(\lambda_{1}^{\prime}\right)$} \\
\hline$\alpha R$ & $\alpha$ & Hopf (1914) & $\begin{array}{c}\text { Gallagher and Mercer } \\
(1962)\end{array}$ & Chebyshev \\
\hline 0.125 & 1. & 3.28 & 3.37 & 3.36944 \\
& 2.5 & 2.22 & 2.19 & 2.19484 \\
1.25 & 1.075 & 3.06 & 3.30 & 3.29666 \\
& 3.225 & 1.88 & 1.90 & 1.90015 \\
12.5 & 2.32 & 2.66 & 2.59 & 2.59078 \\
& 4.64 & 2.07 & 2.11 & 2.11293 \\
\hline
\end{tabular}

TABLE 3. Gallagher and Mercer (1962), Lee and Reynolds (1967) and present Chebyshev results for $\lambda_{\text {; }}$

\begin{tabular}{|c|c|c|c|c|}
\hline \multicolumn{2}{|c|}{} & \multicolumn{3}{|c|}{$\lambda_{i}^{\prime}$} \\
\hline & $\alpha R$ & $\begin{array}{c}\text { Gallagher and Mercer } \\
(1962)\end{array}$ & Lee and Reynolds & Chebyshev \\
\hline$\alpha=0.5$ & 10 & $3.79+0 \imath$ & $3.7921+0 i$ & $3.79209+0 i$ \\
& 50 & $4.82+0 i$ & $4.8232+0 \imath$ & $4.82313+0 i$ \\
& 100 & $10.9+7.45 \imath$ & $10.967+7.379 i$ & $10.9732+7.40239 i$ \\
$\alpha=2.0$ & 20 & $3.16+0 i$ & $3.1644+0 i$ & $3.16446+0 \imath$ \\
& 50 & $7.82+2.41$, & $7.829+2.395 i$ & $7.82578+2.39551 i$ \\
& 200 & $18.9+32.4 i$ & $18.44+32.14 i$ & $18.8721+32.3482 i$ \\
\hline
\end{tabular}


TABLE 4. Ellingsen, Gjevik and Palm (1970) and Chebyshev results for $\operatorname{Im}(c)$

\begin{tabular}{|c|r|c|l|}
\hline \multicolumn{2}{|c|}{} & \multicolumn{2}{|c|}{$-c_{1}$} \\
\hline$\alpha=0.5$ & $\alpha R$ & Ellingsen et al. (1970) & Chebyshev \\
& $2 \times 10^{3}$ & 0.1149 & 0.114515 \\
& $3 \times 10^{3}$ & 0.0899 & 0.0895268 \\
& $5 \times 10^{3}$ & 0.0779 & 0.0776284 \\
$\alpha=1.0$ & $10^{4}$ & 0.0652 & 0.0649455 \\
& $10^{3}$ & 0.0513 & 0.0510825 \\
& $2 \times 10^{3}$ & 0.1196 & 0.119230 \\
& $3 \times 10^{3}$ & 0.0928 & 0.0924906 \\
& $5 \times 10^{3}$ & 0.0802 & 0.0798877 \\
& $10^{4}$ & 0.0668 & 0.0665507 \\
& & 0.0523 & 0.0520923 \\
\hline
\end{tabular}

TABLE 5. Results for $\operatorname{Re}\left(\lambda_{1}^{\prime}\right)$ with $\alpha=2$

\begin{tabular}{|c|c|c|c|c|}
\hline$\alpha R$ & $\operatorname{Re}\left(\lambda_{1}^{\prime}\right)$ & & $\alpha R$ & $\operatorname{Re}\left(\lambda_{1}^{\prime}\right)$ \\
\hline 1000 & 51.6515 & & 70000 & 771.199 \\
2000 & 79.5845 & & 80000 & 841.167 \\
3000 & 102.634 & & 90000 & 908.196 \\
4000 & 123.026 & & 100000 & 972.723 \\
5000 & 141.657 & & 110000 & 1035.08 \\
6000 & 159.001 & & 120000 & 1095.53 \\
7000 & 175348 & & 130000 & 1154.29 \\
8000 & 190.888 & & 140000 & 1211.52 \\
9000 & 205.758 & & 150000 & 1267.36 \\
10000 & 220.057 & & 160000 & 1321.95 \\
20000 & 343.049 & & 170000 & 1375.39 \\
30000 & 445.441 & & 180000 & 1427.79 \\
40000 & 536.445 & & 190000 & 1479.23 \\
50000 & 619.873 & & 200000 & 1529.75 \\
60000 & 697.710 & & & \\
\hline
\end{tabular}

TABLE 6. Values of $\operatorname{Re}\left(\lambda_{1}^{\prime}\right)$ for $\alpha R=10000$

\begin{tabular}{|c|c|c|c|c|}
\hline$\alpha$ & $\operatorname{Re}\left(\lambda_{1}^{\prime}\right)$ & & $\alpha$ & $\operatorname{Re}\left(\lambda_{1}^{\prime}\right)$ \\
\hline $10^{-4}$ & 205.368 & & 60 & 223.154 \\
$10^{-1}$ & 205.434 & & 80 & 221.545 \\
1 & 210.717 & & 100 & 220.838 \\
5 & 244.635 & & 150 & 220.227 \\
10 & 258.896 & & 200 & 220.056 \\
20 & 242.831 & & 500 & 219.928 \\
40 & 227.547 & & 1000 & 219.918 \\
\hline
\end{tabular}


critical Reynolds number, whereby instabilities first appear in the main flow. We have found in theory that plane Couette flow remains stable (or laminar) to very large values of $\alpha R$; that is, the real part of the eigenvalue $\operatorname{Re}\left(\lambda_{1}^{\prime}\right)$ remained positive even for values of $\alpha R$ up to $2 \times 10^{5}$. We note that the values in Table 5, even though always increasing, do have a decreasing increment; that is, the second difference is negative for all values of $\alpha R$ tabulated. However for turbulence we would require $\operatorname{Re}\left(\lambda_{1}^{\prime}\right)<-4 \alpha^{2} / \pi^{2}$, for any particular $\alpha$ and $\alpha R$. Since this has not occurred we can say, therefore, that for $\alpha R<2 \times 10^{5}$ with $\alpha=2$, plane parallel Couette flow as governed by the Orr-Sommerfeld differential equation always remains stable. It is interesting to note also that using the definition of $\lambda_{1}^{\prime}$, we find that the last point in Table 4 gives $\operatorname{Re}\left(\lambda_{1}^{\prime}\right)=200$ approximately which is the same order of magnitude as the result for $\alpha R=10000$ in Table 5. Various other cross correlations are also possible. Table 5 is also in agreement with asymptotic results obtained by Grohne [7] where, despite his erroneous modal crossings (see [6]), he shows that for fixed $\alpha,-c_{t}$ decreases asymptotically towards zero for increasing $R$. The asymptotic results of Davey [1] also confirm this trend.

The effect of maintaining a large fixed $\alpha R$ whilst increasing $\alpha$ is shown in Table 6. We can note that, for increasing $\alpha$, we have a decreasing trend in $\operatorname{Re}\left(\lambda_{1}^{\prime}\right)$ (after $\alpha=10)$. Eventually, however, $\operatorname{Re}\left(\lambda_{1}^{\prime}\right)$ exhibits asymptotic tendencies to a limiting value. For $\alpha R=10000$ the limiting value is given by $\operatorname{Re}\left(\lambda_{1}^{\prime}\right)=219$ approximately.

\section{Conclusions}

The present numerical approach as performed on the Orr-Sommerfeld equation for plane Couette flow has been unable to find a critical Reynolds number beyond which instability in the flow is obtained. This is in conflict with experimental evidence as collected by Reichardt [15] and Robertson [16] who found that turbulence occurred in plane Couette flow for only moderate values of the Reynolds number (600 to 1450). The comment of Deardorff [2] may be valuable in that he points out that in their experiment the critical Reynolds number would depend strongly on the channel entrance conditions which were not adequately taken into account. The inability to find a critical Reynolds number past which turbulence would occur, may also be due in part to the assumption of infinitesimal disturbances which leads to the neglect of all nonlinear (quadratic) terms. Stuart [18, 19], Watson [22] and Ellingsen et al. [3] have examined the effect of these extra nonlinear terms and commented on their destabilizing affects but no definite answer has yet been arrived at. It may well be that plane Couette flow is unstable for finite disturbances, even though it is stable for infinitesimal ones. 


\section{References}

[1] A. Davey, "On the stability of plane Couette flow to infinitesimal disturbances", J. Flutd Mech. 57 (1973), 369-380.

[2] J. W. Deardorff, "On the stability of viscous plane Couette flow", J. Fluld Mech. 15 (1963), 623-631.

[3] T. Ellingsen, B. Gjevik and E. Palm, "On the nonlinear stability of plane Couette flow", $J$. Fluid Mech. 40 (1970), 97-112.

[4] J. G. F. Francis, “The QR transformation, a unitary analogue to the LR transformation", Parts 1 and 2, Comput. J. 4 (1961), 265-271, 332-345.

[5] A. P. Gallagher and A. McD. Mercer, "On the behaviour of small disturbances in plane Couette flow", J. Fluid Mech. 13 (1962), 91-100.

[6] A. P. Gallagher and A. McD. Mercer, "On the behaviour of small disturbances in plane Couette flow. Part 2. The higher eigenvalues", J. Fluid Mech. 18 (1964), 350-352.

[7] D. Grohne, "Über das Spektrum bei Eigenschwingungen ebener Laminarströmungen", $Z$. Angew. Math. Mech. 34 (1954), 344-357 (Translated as "On the spectrum of natural oscillations of two-dimensional laminar flows", Tech Memor. N.A.C.A. Wash. No. 1417).

[8] L. Hopf, "Decrement of small vibrations in the flow of a viscous fluid", Ann. Physik 44 (1914), $1-60$.

[9] R. Jordinson, "The flat plate boundary layer, Part 1: Numerical integration of the OrrSommerfeld equation", J. Fluld Mech. 43 (1970), 801-811.

[10] L. H. Lee and W. C. Reynolds, "On the approximate and numerical solution of Orr-Sommerfeld problems", Quart. J. Mech. Appl. Math. 20 (1967), 1-22.

[11] A. G. Morris, "Eigenvalues by numerical methods", Ph.D. Thesis, Wollongong University College, University of New South Wales, 1973.

[12] A. G. Morris and T. S. Horner, "Chebyshev polynomials in the numerical solution of differential equations", Math. Comp. 31 (1977), 881-891.

[13] S A. Orszag, "Accurate solution of the Orr-Sommerfeld stability equation", J. Fluid Mech. 50 (1971), 689-703.

[14] M. R. Osborne, "Numencal methods for hydrodynamic stability problems", SIAM J. Appl. Math. 15 (1967), 539-557.

[15] H. Reichardt, "Gesetzmässigkeiten der geradlinigen turbulenten Couetteströmung", Mittellungen aus dem Max-Planck Instttut für Strömungsforschung und der Aerodynamischen Versuchsanstalt 22 (1959).

[16] J. M. Robertson, "On turbulent plane-Couette flow", Proc. 6th Midwestern Conference on Fluld Mechanics (1959), 169-182.

[17] H. B. Squire, "On the stability of the three-dimensional disturbances of viscous flow between parallel walls", Proc. Roy. Soc. London Ser. A 142(1933), 621-628.

[18] J. T. Stuart, "On the nonlinear mechanics of hydrodynamic stability", J. Fluid Mech. 4 (1958), $1-21$.

[19] J. T Stuart, "On nonlinear mechanics of wave disturbances in stable and unstable parallel flows. Part 1. The basic behaviour in plane Poiseuille flow", J. Fluid Mech. 9 (1960), 353-370.

[20] L. H. Thomas, "Stability of plane Poiseuille flow", Phys. Rev. 91 (1953), 780-783.

[21] Th. L. van Stijn and A. I. van de Vooren, "An accurate method for solving the Orr-Sommerfeld equation", J. Engrg. Math. 14 (1980), 17-26.

[22] J. Watson, "On the non-linear mechanics of wave disturbances in parallel flows. Part 2. The development of a solution for plane Poiseuille flow and for plane Couette flow", J. Flutd Mech. 9 (1960), 371-389.

[23] J. H. Wilkinson, The algebraic eigenvalue problem (Oxford University Press, London, 1965) 